\title{
LA UNIVERSIDAD DE SEVILLA Y SU ÁMBITO GEOGRÁFICO DE ATRACCIÓN*
}

\author{
Jesús VENTURA FERNÁNDEZ**
}

\section{INTRODUCCIÓN A LA UNIVERSIDAD HISPALENSE}

La Universidad de Sevilla, la más antigua de todas las andaluzas (Colegio Santa María de Jesús fundado por Maese Rodrigo Fernández de Santaella a finales deI s. XV y bula del Papa Julio II en 1505 por la que se otorgaba al mismo la facultad de conferir grados en Teología, Filosofía, Derecho, Medicina y Arte), forma parte de la red de universidades con mayor tradición de todo el Estado. Su acelerado proceso de crecimiento, tanto de centros, como de profesores y sobre todo alumnos, ha conllevado una reordenación administrativa mediante la cual distintas provincias se ha ido desgajando de su distrito universitario (que correspondía antaño a las cuatro más occidentales de Andalucía -Sevilla, Huelva, Cádiz y Córdoba-, más Badajoz en Extremadura).

Trataremos a continuación de analizar la procedencia estudiantil en un momento clave para la historia de esta institución superior: poco después de que se segregara su última provincia foránea (Huelva), y poco antes de que se activara un proceso de desconcentración intemo, con la potenciación de nuevos centros en la villa ducal de Osuna (con antigua Universidad menor), así como la aparición de una segunda universidad pública en la capital andaluza (bajo el nombre del ilustrado Pablo de Olavide), estando incluso previsto a medio plazo la constitución de una universidad privada en el entomo metropolitano de Sevilla.

Así pues diremos en primer lugar que la Hispalense impartió docencia durante el curso 92/93 (último en el que su distrito comprendía también la provincia de Huelva) a un total de 68.354 alumnos (I.E.A., 1994) (la abrumadura mayoría de

* Trabajo basado en una parte de la Tesis doctoral defendida en la Universidad de Sevilla con la máxima calificación durante el curso 95/96, titulada Los servicios educativos en Andalucía: territorio y planificación sectorial, y dirigida por el Dr. Cano García, Catedrático de Análisis Geográfico Regional de la Hispalense, con la que el autor obtuyo el Premio Extraordinario de Doctorado de dicho año.

** Profesor Asociado de Análisis Geográfico Regional de la Universidad de Sevilla. 
ellos -más del $90 \%$ - en los centros sevillanos, según puede deducirse de los datos del Octavo Mapa Escolar de Andalucía para el curso 1991/92. Este contingente es el resultado de un notable proceso de crecimiento en el que podemos dejat constancia de algunas cifras puntuales.

En 1977 el número de alumnos se elevaba a 35.005 contabilizando los centros de Cádiz, todavía dependientes de la Hispalense (FERNANDEZ ARUFE, 1982), mientras que en el curso 86/87 ascendía a 41.681 (UNIVERSIDAD HISPALENSE, 1988 ), sin contabilizar en este último caso las 4.781 matrículas del Instituto de Idiomas, pero incluyendo las correspondientes a los centros de Huelva. Como vemos el ritmo de crecimiento era interesante pero contenido, y sin embargo éste se ha disparado de manera evidente en los últimos años, hasta el punto de que, en un estudio dedicado a La situación urbanística de la Universidad de Sevilla (CLAVE S.L., 1990), la hipótesis media situaba el volumen de matrículas para el año 2000 en 55.860, cantidad rebasada con claridad casi una década antes (56.918 según el Anuario Estadístico Andalucía-1991; I.E.A., 1992).

Рara llevar a cabo esta ingente tarea de enseñanza -así como la investigadora que también le es propia-, la Universidad de Sevilla ha visto pasar su presupuesto de 6.625 millones de pesetas en 1985 (UNIVERSIDAD HISPALENSE, 1987) a los 22.221 de 1993 (UNIVERSIDAD DE SEVILLA, 1994) (incremento del 235 $\%$ en 8 años, sin considerar que los gastos reales de este último ejercicio se elevaron finalmente hasta los 31.781 millones; UNIVERSIDAD DE SEVILLA, 1995).

Esto, sin embargo, no le ha conferido un gasto por alumno próximo a la media de las universidades andaluzas, sino que éste es inferior en más de un $14 \%$. Además la situación se agrava de manera evidente si atendemos a la relación de inversiones reales por alumno, de sólo el $16,90 \%$ respecto a la media regional, según datos de HERNANDEZ, LASSIBILLE y NAVARRO (1993) para pesetas corrientes de 1992. No obstante este problema se ha pretendido mejorar con presupuestos como los de 1994, en el que esta última partida se incrementó hasta los 4.997 millones, alcanzando el 19,34 \% del total de los gastos frente al 6,33\% del año anterior (si bien en la práctica éste llegó al 18,29 \% debido a diversas modificaciones presupuestarias) (UNIVERSIDAD DE SEVILLA, 1994 y 1995).

Por otro lado debemos decir también que en el informe urbanístico de CLAVE S.L. (I990) se abunda como es lógico en la distribución espacial del alumnado por los distintos centros propios de la Universidad en Sevilla, que alcanzaban la cifra de 26 , pero que se agrupan o se localizan de forma individualizada en 8 ámbitos diferentes según apreciamos en el Gráfico $n^{\circ} 1$, con cuantía de matrícula presente (por 1998) y prevista muy dispar entre ellos.

Esto determina igualmente la aparición de una serie de flujos principales (de más de 1.000 desplazamientos diarios durante el período de clases) entre distintas partes de la ciudad y diversos conjuntos de centros universitarios, como puede observarse en el Gráfico $n^{\circ}$ 2. En esta última figura destaca el poder de atracción 
GRÁFICO № 1

NÚMERO DE ALUMNOS POR CONJUNTOS DE CENTROS

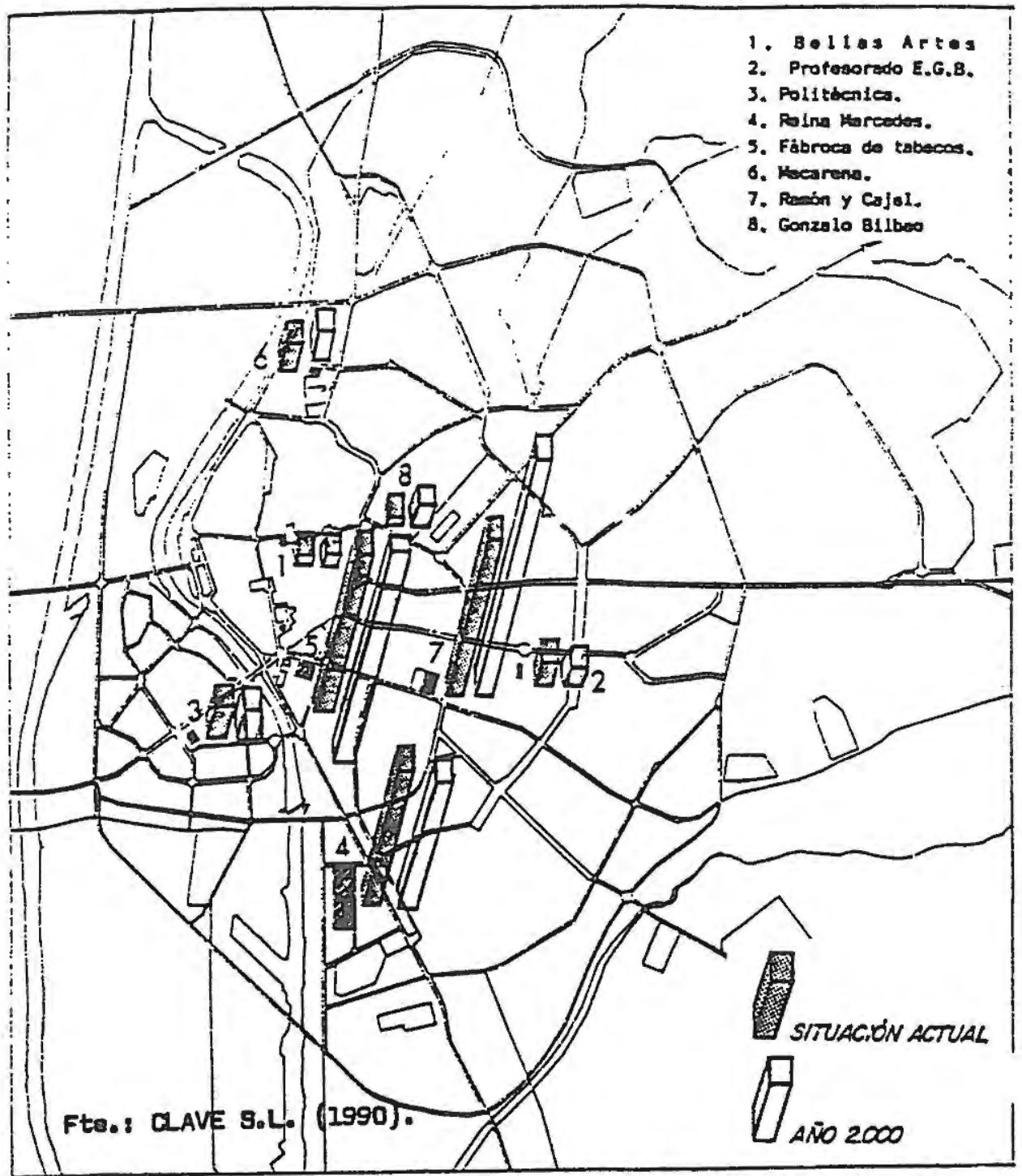


GRÁFICO № 2

FLUJOS PRINCIPALES (MÁS DE 1.000) ENTRE ZONAS Y CONJUNTOS DE CENTROS

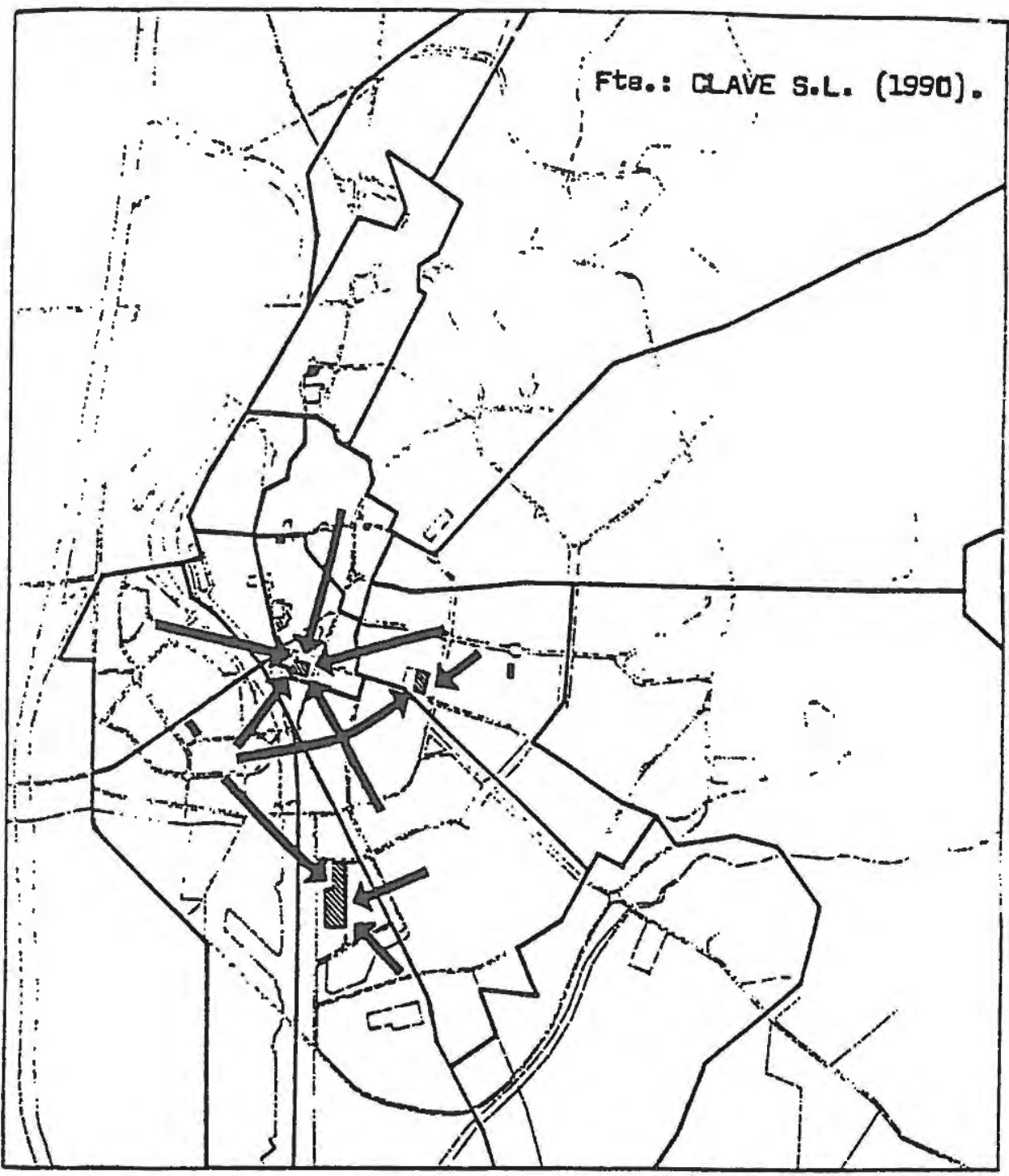


de las zonas de Fábrica de Tabacos (donde se imparte Derecho, Filología, y Geografía e Historia), Reina Mercedes (con las facultades de Ciencias y escuelas técnicas), y Ramón y Cajal (Ciencias Económicas y Empresariales, así como Filosofía y Sicología).

\section{ESTUDIO DE UN CASO CONCRETO: LA FACULTAD DE GEOGRAFÍA E HISTORIA}

Ahora bien, dado el enfoque del estudio que ahora estamos realizando, más nos interesa aquí la procedencia geográfica del alumnado hacia esta función docente superior ofertada de manera específica por la ciudad, pero cuya demanda desborda obviamente los límites urbanos e incluso los de su entomo más próximo. Para ello lo primero que hicimos fue acudir a un centro consolidado y que entendemos puede servir como ejemplo. Nos referimos a la Facultad de Geografía e Historia, que, con una matrícula que en la última década osciló entre los 2.588 alumnos del curso $85 / 86$ y los 2.032 del $90 / 91$, ha venido a suponer entre el $7,77 \%$ y el $3,49 \%$ de los alumnos de la Universidad de Sevilla (en 1984 y 1992 respectivamente).

No obstante cabría comentar que en los años más recientes se observa un significativo aumento de las inscripciones a raíz de la implantación de los nuevos planes de estudio (en especial de la titulación en Antropología Social y Cultural -única de $2^{\circ}$ Ciclo existente en la actualidad en Andalucía-), así como debido a un resurgir del interés por las disciplinas humanísticas en general y de las ciencias sociales en particular, lo que nos conduce a valores próximos a los 4.000 alumnos en el curso 95/96 (3.415 de los cuales hemos podido considerar para nuestro estudio), y a una participación que supera de nuevo el $5 \%$ del total.

En todo caso lo que más nos interesa es que debido a la informatización de la matrícula se ha podido acceder a algunos datos de evidente orientación geográfica. En concreto es factible obtener la provincia de nacimiento de los alumnos, así como la de residencia durante el curso, mientras que la provincia de residencia familiar (también requerida en el impreso de matriculación) no está disponible en la aplicación informática actualmente vigente. No obstante pensamos que pueden deducirse algunas cuestiones.

De este modo, una vez agregadas en dos únicos listados las 10 series que nos fueron facilitadas por la Secretaría del Centro (una para cada una de las nuevas titulaciones en Geografía, Historia, Historia del Arte, y Antropología, más la de los alumnos del plan antiguo de Geografía e Historia, según las dos variables antes mencionadas), nos hemos decidido por presentar mediante Cuadros los datos agrupados por CC.AA. y a través de Mapas los relativos al escalón provincial.

En el primero de estos cuadros destaca -aparte como es lógico de una abrumadura mayoría de andaluces- el origen natalicio de los alumnos de este cen- 
tro en todas las CC.AA. españolas más las ciudades autónomas de Ceuta y Melilla así como una proporción significativa $(1,87 \%)$ de nacidos en el extranjero. Sir embargo no podemos olvidar que en este hecho influye una cuestión referida : nuestra historia socio-económica reciente como es la emigración andaluza a otra: regiones más desarrolladas del Estado e incluso al extranjero durante los años 6( sobre todo, lo que ha determinado que muchos de los hijos de los afectados po estos procesos migratorios, y en función de los fenómenos de retorno, sean ahor: estudiantes en nuestras universidades. Esto puede apreciarse en los datos corres pondientes a Cataluña y Madrid, entre el 2,25 y el 2,98 \% del total, e incluso, muy posiblemente, en el porcentaje de nacidos fuera de España.

Ahora bien, en el caso extremeño (con el 3,71 \% de la matrícula) sí creemo: que debe pesar una cierta dependencia funcional de la Universidad sevillana, puestc que el centro equivalente al que estamos ahora analizando se localiza en Cáceres lo que es probable que determine una atracción hacia Sevilla de los alumnos inte. resados por estos estudios en la provincia de Badajoz, históricamente inserta en e distrito sevillano hasta la creación de la Universidad de Extremadura en 1973

\section{CUADRO I}

DISTRIBUCIÓN POR CC.AA. DEL LUGAR DE NACIMIENTO DE LOS ALUMNOS DE LA FACULTAD DE GEOGRAFÍA E HISTORIA (CURSO 1995/96)

\begin{tabular}{|c|c|c|}
\hline CC.AA. y otros ámbitos & № alumnos & $\%$ \\
\hline 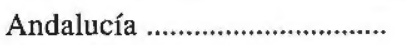 & 2.824 & 82,69 \\
\hline 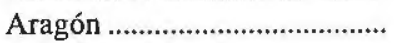 & 16 & 0,46 \\
\hline 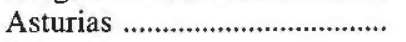 & 5 & 0,14 \\
\hline 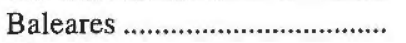 & 8 & 0,23 \\
\hline 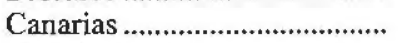 & 12 & 0,35 \\
\hline 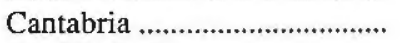 & 4 & 0,11 \\
\hline 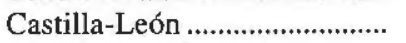 & 33 & 0,96 \\
\hline Castilla-La Mancha ................. & 31 & 0,90 \\
\hline 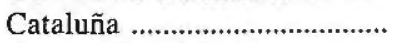 & 77 & 2,25 \\
\hline Comunidad Valenciana ............ & 21 & 0,61 \\
\hline 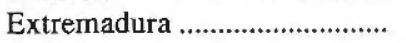 & 127 & 3,71 \\
\hline 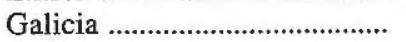 & 11 & 0,32 \\
\hline 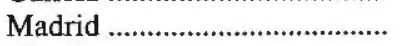 & 102 & 2,98 \\
\hline 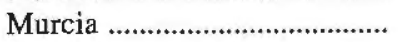 & 8 & 0,23 \\
\hline 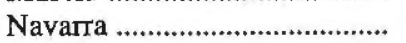 & 3 & 0,08 \\
\hline 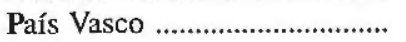 & 22 & 0,64 \\
\hline La Rioja & 5 & 0,14 \\
\hline Ceuta y Melilla ........................... & 9 & 0,26 \\
\hline 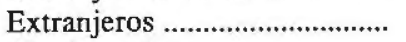 & 64 & 1,87 \\
\hline 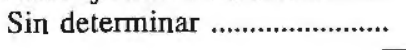 & 33 & 0,96 \\
\hline 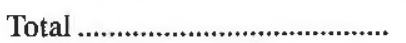 & 3.415 & 100,00 \\
\hline
\end{tabular}

Fuente: Elaboración propia a partir de datos facilitados por la Secretaría de la Facultad de Geografía e Historia 
MAPA 1

PROVINCIAS DE NACIMIENTO DEL ALUMNADO.

FACULTAD DE GEOGRAFÍA E HISTORIA. CURSO 1995/96

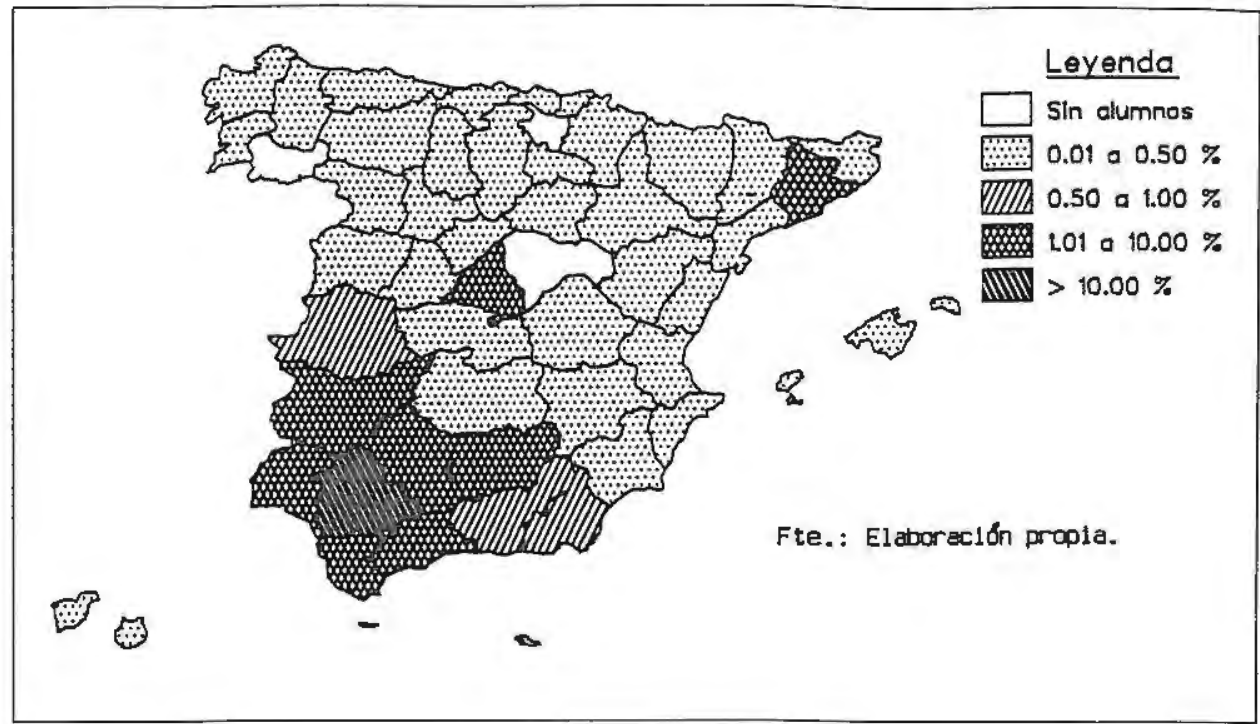

MAPA 2

PROVINCIAS DE RESIDENCIA DURANTE EL CURSO.

FACULTAD DE GEOGRAFÍA E HISTORIA. CURSO 1995/96

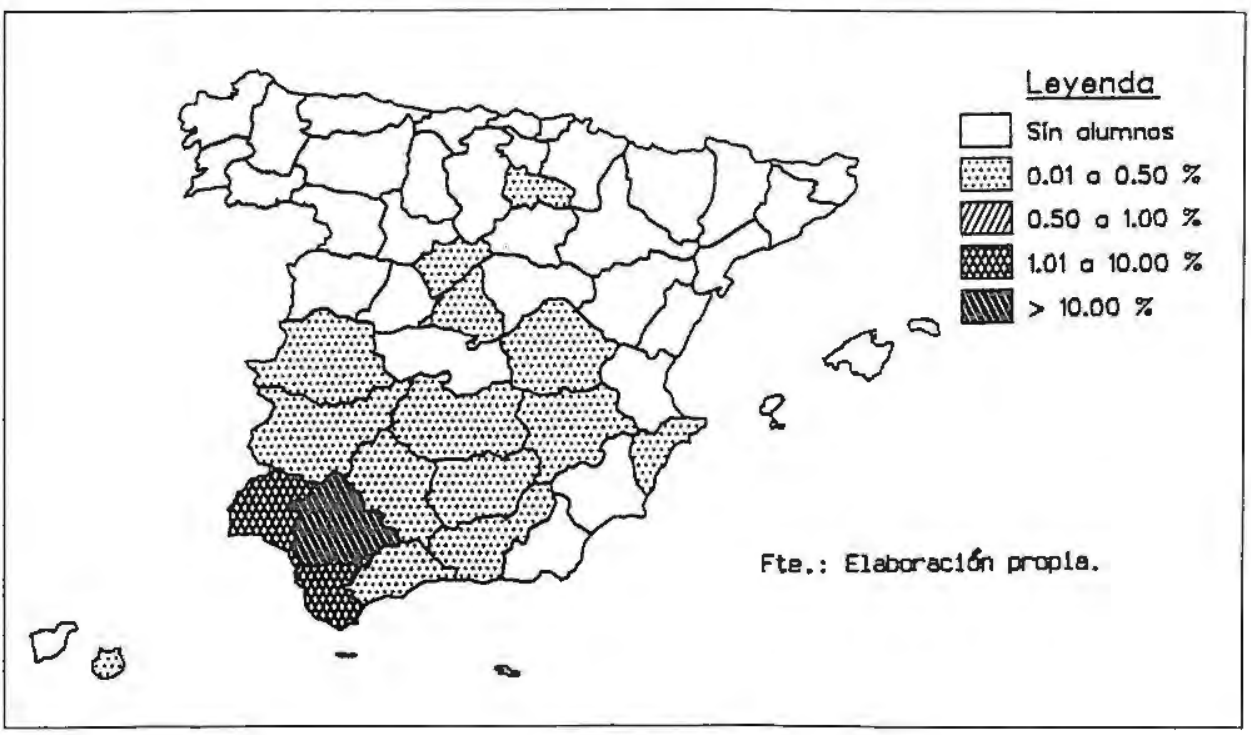


Por su parte la representación espacial provincializada del origen natalicio de los alumnos de esta Facultad nos habla de que sólo no están recogidos 3 de los 52 casos posibles (la red de 50 provincias más Ceuta y Melilla); tratándose en concreto de las entidades de Alava, Guadalajara y Orense. De todos modos podemos destacar evidentemente la concentración en las andaluzas, en especial en las occidentales, que han tenido de manera tradicional una mayor vinculación con la Hispalense (los nacidos en Sevilla suponen el 62,48 \%, los gaditanos el 7,23\%, los onubenses el 5,97, y los cordobeses 2,54), mientras que en el resto de la región encontramos valores poco significativos que oscilan entre el 0,58 \% de Almería y el 1,69 \% de Málaga. Respecto a los casos extrarregionales resaltar a Badajoz, Madrid, Barcelona y a los nacidos en el extranjero, según los motivos anteriores.

En relación con la provincia de residencia durante el curso, de menor interés para nosotros, debemos decir que en esta variable sobresale el alto porcentaje de personas que desconocía la misma en el momento de realizar su matrícula (en tomo al $32 \%$ ), lo cual no deja de ser algo relativamente lógico, sobre todo para los alumnos procedentes de fuera de la ciudad. Lo que no parece tan razonable es que en este listado surgan residencias durante el año académico en Comunidades como Canarias, Castilla-León, Valencia o la Rioja, por poner los ejemplos más llamativos. Todo ello nos habla de posibles errores de interpretación a la hora de cumplimentar el impreso por parte de los interesados, confundiéndose el domicilio familiar con el de residencia durante el curso, aunque también puede deberse a casos específicos de alumnos con matrícula libre, especialmente en la titulación de Antropología, cuya área de atracción es amplia al contar con muy escasa competencia en el mapa de titulaciones del Estado. Así pues pensamos que sería más atractivo el disponer de la provincia de residencia familiar habitual según haremos para el estudio general de la Universidad.

De cualquier modo puede comentarse la lógica concentración de la residencia en Andalucía, con el 67,58 \%, sobre todo en la provincia de Sevilla (64,50\%), situándose a continuación Cádiz y Huelva, y no apareciendo el caso almeriense. Por último, aunque sea a título casi complementario, incluimos un Cuadro equivalente al anterior para los datos de las CC.AA. de residencia durante el curso.

\section{EL ÁREA DE INFLUENCIA DE LA UNIVERSIDAD DE SEVILLA}

A nivel de Universidad de Sevilla se nos informó en el Centro de Proceso de Datos (C.P.D.), como organismo encargado -entre otras cuestiones- del tratamiento de la matrícula, que ésta no se encontraba informatizada para todos los centros según hemos visto en el caso de la Facultad de Geografía e Historia, sino que todavía existía una serie de ellos que no aplicaban el Sistema Gestor de Base de Datos 
CUADRO II

DISTRIBUCIÓN POR CC.AA. DEL LUGAR DE RESIDENCIA DURANTE EL CURSO DE LOS ALUMNOS DE LA FACULTAD DE GEOGRAFÍA E HISTORIA (CURSO 1995/96)

\begin{tabular}{|c|c|c|}
\hline CC.AA. afectadas & № Alumnos & $\%$ \\
\hline 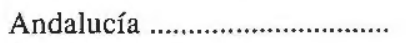 & 2.308 & 67,58 \\
\hline 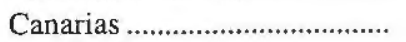 & 1 & 0,02 \\
\hline 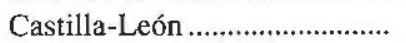 & 1 & 0,02 \\
\hline Castilla-La Mancha ................. & 4 & 0,11 \\
\hline Comunidad Valenciana ........... & 1 & 0,02 \\
\hline 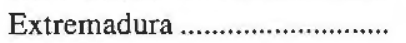 & 5 & 0,14 \\
\hline 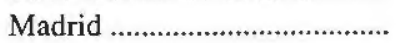 & 1 & 0,02 \\
\hline 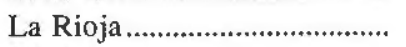 & 1 & 0,02 \\
\hline 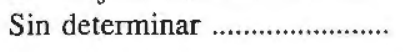 & 1.093 & 32,00 \\
\hline 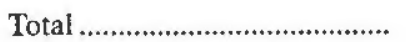 & 3.415 & 100,00 \\
\hline
\end{tabular}

Fuente: Elaboración propia a partir de datos facilitados por la Secretaría de la Facultad de Geografía e Historia.

ORACLE, que es el que ha permitido todo el manejo anterior. Estos eran en concreto los casos de los centros del área de la Salud (facultades de Medicina y Odontología y la E.U. de Ciencias de la Salud), así como la Facultad de CC. de la Información y las escuelas universitarias de ingenieros técnicos agrícolas y la de Trabajo Social.

No obstante, para cubrir los requerimientos estadísticos, se encarga anualmente a una empresa privada la introducción de datos relativa a todos los alumnos matriculados (incluidos obviamente los correspondientes a centros externos a la red ORACLE), aunque su fiabilidad es menor a la del sistema informático propio de la Universidad, pues en éste los datos se confrontan con el alumno en el mismo momento de la matrícula. De todos modos dicho mecanismo, que podemos calificar de circunstancialmente extraordinario, debe servirnos para realizar una aproximación al ámbito de atracción general de la Universidad de Sevilla. En esta ocasión los datos corresponden al curso anterior 94/95 (aún no se disponía de los más recientes), si bien se ha conseguido que se refieran a la localidad de residencia familiar habitual según su Código Postal (uno de los casilleros en el que al parecer se pone mayor énfasis para que sea cumplimentado por parte del alumno), lo cual es de gran interés para nosotros. Tal y como hicimos en el ejemplo particular que analizábamos antes, presentamos a continuación Cuadro con el desglose por CC.AA., y Mapa con el detalle de la distribución provincial.

Como era de esperar tampoco en esta ocasión aparece Comunidad Autónoma alguna que no se vea afectada, aunque sea mínimamente, por el ámbito de atracción de la Universidad de Sevilla según el lugar de residencia de la familia del 


\section{CUADRO III}

DISTRIBUCIÓN POR CC.AA. DE LA PROCEDENCIA GEOGRÁFICA DEL ALUMNADO DE LA UNIVERSIDAD DE SEVILLA SEGÚN EL LUGAR DE RESIDENCIA FAMILIAR (CURSO 1994/95)

\begin{tabular}{|c|c|c|}
\hline CC.AA. y otros ámbitos & № alumnos & $\%$ \\
\hline 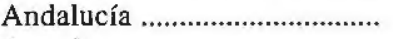 & 61.579 & 90,58 \\
\hline Aragón & 23 & 0,03 \\
\hline 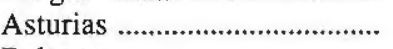 & 17 & 0,02 \\
\hline 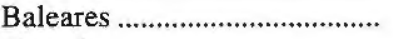 & 32 & 0,04 \\
\hline 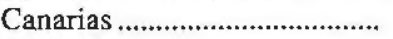 & 125 & 0,18 \\
\hline 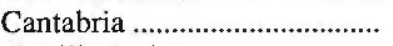 & 20 & 0,02 \\
\hline 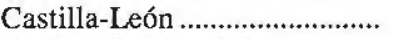 & 56 & 0,08 \\
\hline Castilla-La Mancha .................... & 193 & 0,28 \\
\hline 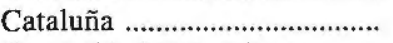 & 35 & 0,05 \\
\hline Comunidad Valenciana ............ & 43 & 0,06 \\
\hline 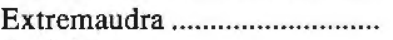 & 1.666 & 2,45 \\
\hline 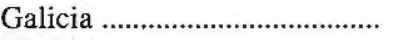 & 25 & 0,03 \\
\hline 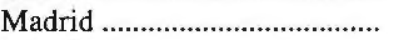 & 114 & 0,16 \\
\hline 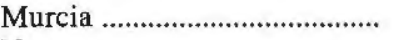 & 42 & 0,06 \\
\hline 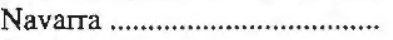 & 6 & 0,008 \\
\hline 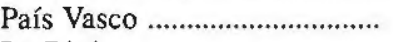 & 28 & 0,04 \\
\hline 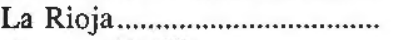 & 4 & 0,005 \\
\hline Ceuta y Melilla ........................... & 257 & 0,37 \\
\hline 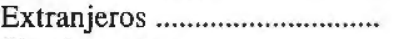 & 145 & 0,21 \\
\hline 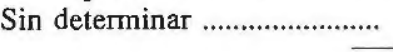 & 3.572 & 5,25 \\
\hline Total & 67.982 & 100,00 \\
\hline
\end{tabular}

Fuente: Elaboración propia a partir de los datos suministrados por el C.P.D. de la Universidad de Sevilla.

CUADRO IV

REPARTO ENTRE LAS PROVINCIAS ANDALUZAS DE LOS ESTUDIANTES DE LA UNIVERSIDAD DE SEVILLA (CURSO 1994/95)

\begin{tabular}{|c|c|c|c|}
\hline Provincia & № alumnos & $\% /$ And. & $\% /$ Total \\
\hline Almería ..................... & 317 & 0,51 & 0,46 \\
\hline 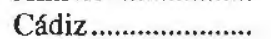 & 4.897 & 7,95 & 7,20 \\
\hline Córdoba ......................... & 1.644 & 2,66 & 2,41 \\
\hline Granada ..................... & 353 & 0,57 & 0,51 \\
\hline 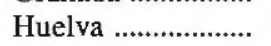 & 3.851 & 6,25 & 5,66 \\
\hline 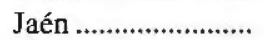 & 638 & 1,03 & 0,93 \\
\hline Málaga ......................... & 957 & 1,55 & 1,40 \\
\hline 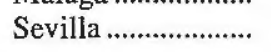 & 48.922 & 79,44 & 71,96 \\
\hline Andalucía ................. & 61.579 & 100,00 & 90,58 \\
\hline
\end{tabular}

Fuente: Elaboración propia a partir de los datos suministrados por el C.P.D. de la Universidad de Sevilla, 
alumno, y que podemos identificar con su origen geográfico. De hecho sólo hay una provincia española (Lugo) de la que "no era" ningún alumno de la Hispalense durante el curso 94/95. Ahora bien, únicamente el porcentaje de extremeños es significativo $(2,45)$, por lo que se acentúa respecto al caso particular anterior una procedencia estrictamente andaluza. Es por ello que exponemos a continuación, dada su importancia, el reparto entre las 8 provincias de la región.

Según puede apreciarse, incluso dentro de Andalucía, se produce como es obvio una clarísima polarización en tomo a la provincia que da nombre a la Universidad, pues Sevilla aglutina a casi el $80 \%$ de los estudiantes andaluces de la Hispalense. A gran distancia se sitúan Cádiz y Huelva primero, y Córdoba, Málaga, Jaén, Granada y Almería por ese orden después, prelación en la que parece que existe un cierto peso de la historia universitaria andaluza (sobre todo si tenemos en cuenta que Badajoz tiene también -según se observa en el Mapa siguiente- un comportamiento similar), aunque es evidente que en ello influye la mera proximidad geográfica. En efecto, comprobamos cómo son las 5 provincias limítrofes con Sevilla las únicas que se sitúan en el intervalo inmediatamente anterior al superior, en el que se posiciona el enclave sevillano. Habría sólo dos casos en un nivel intermedio (Jaén y Granada), puesto que incluso Almería queda englobada con un resto de provincias en las que la dependencia es simplemente anecdótica (inferior al 0,5\% de la matrícula). Además hemos representado asímismo el área de influencia de la Facultad de Geografía e Historia para el curso 94/95 (con datos sólo en los antiguos planes de estudio: desde $2^{\circ}$ Curso en dicha fecha), según la misma variable que en el caso general (residencia familiar), y lo que se aprecia es un debilitamiento de esta atracción específica: muchas provincias sin presencia de alumnos, Jaén y Granada pasan al nivel más bajo, e incluso Málaga deja de estar por encima de la unidad porcentual (0,55 en concreto), lo que entendemos se debe a una profusa difusión territorial en la oferta de este tipo de estudios humanísticos.

Por último, dado el peso específico de la provincia de Sevilla entre el alumnado de esta Universidad (en torno al $72 \%$ del total), hemos aumentado la escala de trabajo y reflejamos a continuación el reparto territorial de dicha matrícula, inicialmente en valores absolutos, optándose además por su representación mediante densidad de puntos, ya que ésta resulta bastante elocuente. De esta manera detectamos una clarísima concentración de individuos en la aglomeración urbana de Sevilla, pero también en otras zonas significativas, como son gran parte de la Campiña sevillana, de la comarca del Aljarafe, y de la Vega del Guadalquivir, así como en torno al enclave de Estepa. En el resto de la provincia los valores disminuyen con bastante claridad.

A continuación hemos cartografiado, para concluir, la distribución espacial de la proporción de alumnos universitarios en Sevilla durante el curso 94/95, sobre el total de cada población municipal de derecho a 1 de enero de 1994 (con la excepción del caso de Umbrete, en el que hemos tenido que acudir a la del año 
MAPA 3

PROVINCIAS DE PROCEDENCIA DEL ALUMNADO. UNIVERSIDAD DE SEVILLA. CURSO 1994/95

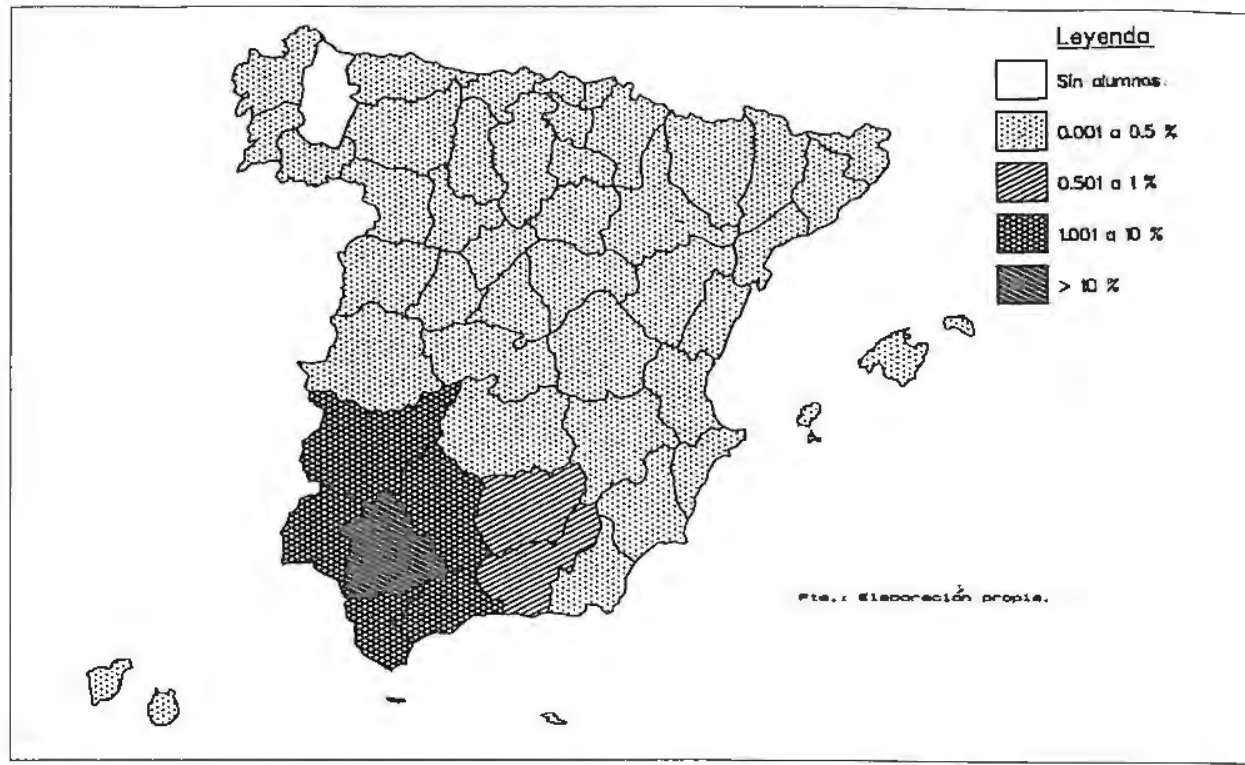

MAPA 4

PROVINCIAS DE PROCEDENCIA DEL ALUMNADO.

FACULTAD DE GEOGRAFÍA E HISTORIA. CURSO 1994/95

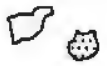

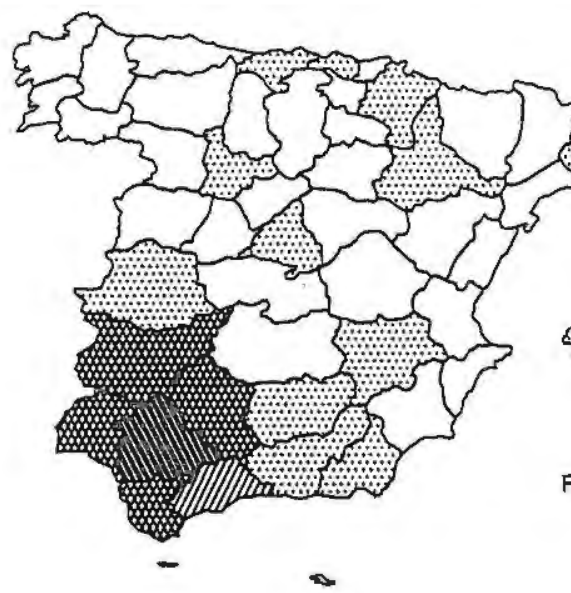

Fte.: Elaborac16́n propla. 


\section{MAPA 5}

MUNICIPIOS SEVILLANOS DE PROCEDENCIA DEL ALUMNADO. UNIVERSIDAD DE SEVILLA. CURSO 1994/95

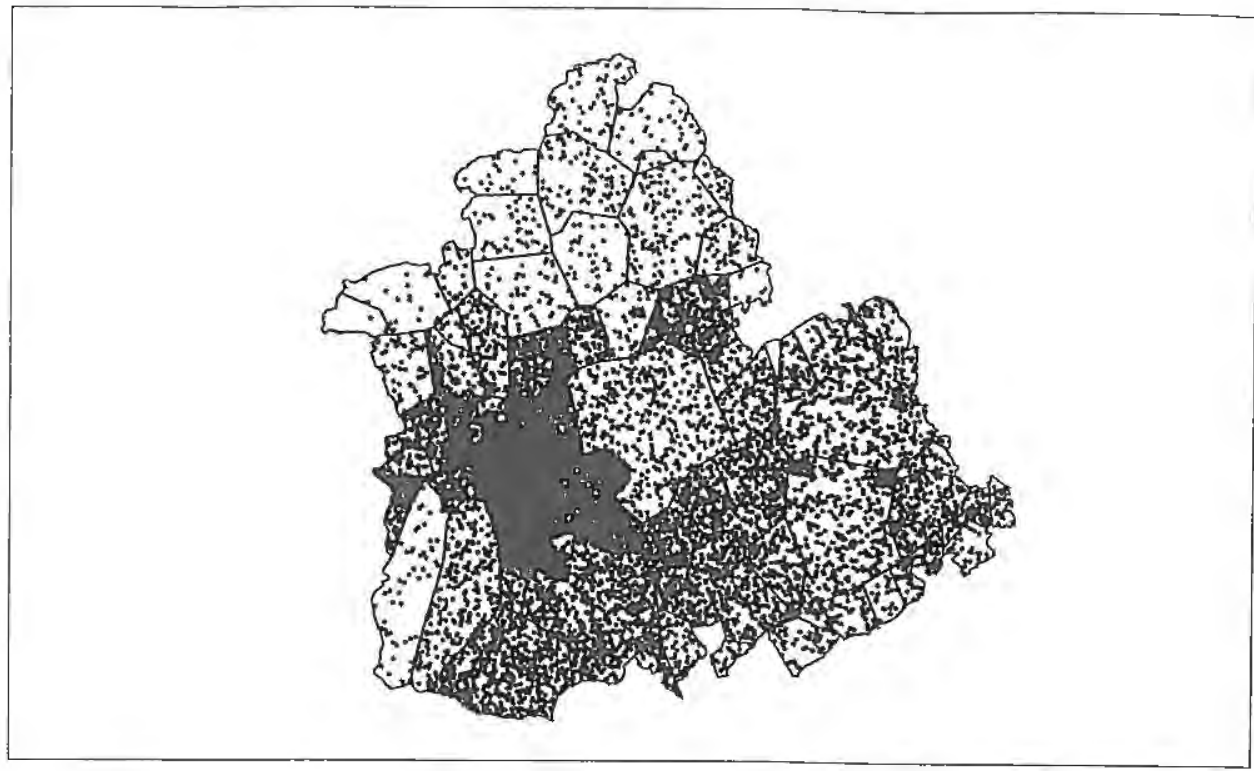

MAPA 6

TASA DE ESTUDIANTES UNIVERSITARIOS EN LA PROVINCIA DE SEVILLA. UNIVERSIDAD DE SEVILLA. CURSO 1994/95

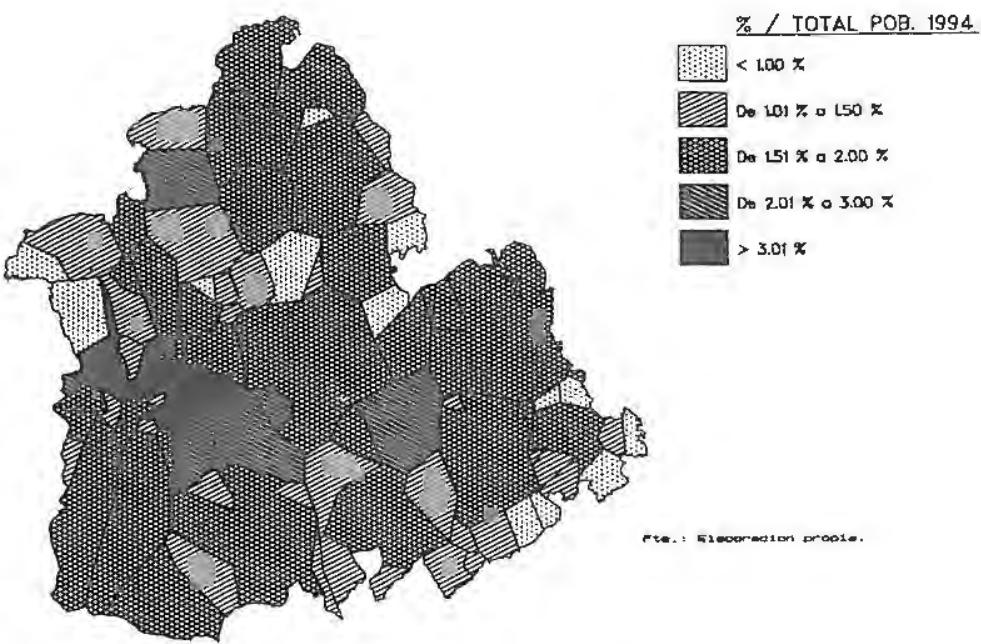


anterior). Esto ha sido así ya que carecemos de un desglose por edades que no acercara a una tasa más ajustada y significativa. El recorrido de esta variable osci la desde el 0,24 \% de Peñaflor (en el límite de la provincia de Sevilla con la dı Córdoba, por lo que pensamos puede producirse una atracción hacia esta otri Universidad), hasta el $15,26 \%$ de Castilleja de Guzmán, muy condicionado por sı escasa población absoluta (sólo 583 habitantes en dicho año), y su carácter dı municipio residencial próximo a la capital.

En el resto del territorio apreciamos una serie de orlas alrededor de la ciudar central, en este caso Sevilla: por un lado el término principal y su entorno metro politano, con valores superiores al 2 e incluso al $3 \%$ de su población total; a con tinuación las zonas de Campiña, Vegas Bajas y Marisma, e incluso una gran partı de la Sierra Norte, con datos entre el 1,5 y el $2 \%$; y por último una periferia pro vincial -en especial la deprimida Sierra Sur o Subbético sevillano- con cifras es casas, en ocasiones inferiores al $1 \%$ de sus habitantes. Dos excepciones a este esquema general son los casos de Almadén de la Plata y Marchena, con valore: por encima de lo esperado, entre el 2 y el $3 \%$.

\section{A MODO DE COLOFÓN}

A lo largo de las páginas anteriores hemos pretendido, aplicando un método d trabajo evidentemente inductivo, desentrañar cuál es el ámbito de atracción de 1: Universidad de Sevilla en un momento que, según decíamos al principio, entende mos como decisivo dentro de su ya dilatado discurrir histórico. En la evolución d la Universidad española el incremento en el acceso a la enseñanza superior h: originado un proceso de descentralización administrativa mediante el cual se har creado nuevas de estas instituciones - tanto públicas como algunas privadas- en e conjunto del Estado, hasta alcanzar una cifra que supera ya el medio centenar. Sir embargo esto no ha supuesto, de momento, un reparto equitativo de la demand: estudiantil, que continúa muy concentrada en algunas de ellas -como sería l: Hispalense-, lo que entendemos obedece a un conjunto de razones.

Por un lado se debe al mero dato geográfico de que las universidades tradicio nales coinciden por regla general con ámbitos poblacionales bastante densos, y po otro está en relación con el hecho funcional de que las nuevas no siempre cuentar con el abanico de titulaciones más adecuado a sus necesidades reales, con las d mayor demanda, o simplemente con algunas de ellas. Enmedio de ambos tipos d circunstancias se sitúan además motivos de carácter más bien subjetivo, como sor los vinculados a la tradición y al prestigio adquirido a través del tiempo, o simple mente un deseo juvenil de salir del entorno local y del control familiar, lo qur también juega en favor de las ciudades universitarias tradicionales.

Este problema se intenta resolver en la actualidad a través de la desconcentraciór de centros (potenciando por ejemplo estudios superiores en determinadas cabece 
ras comarcales como en nuestro caso sería Osuna), así como con la constitución de nuevas universidades en las localidades centrales. Aunque todos estos procedimientos son cuestionables, y no son pocos quienes se posicionan a favor de ayudas a la movilidad estudiantil en lugar de la constitución -siempre costosa- de nuevas y pequeñas instituciones, lo cierto es que debemos asumirlos como consustanciales al modelo que finalmente se ha impuesto, en gran medida por su gran contenido político.

Es por ello el que entendamos que, en todo caso, el seguimiento cronológico al ámbito de atracción de las distintas universidades (e incluso de sus diversos centros y titulaciones) se manifiesta como indispensable para constatar la correcta marcha de estos mecanismos de descongestión educativa, con los cuales se pretende, en principio, racionalizar y optimizar el importante gasto público que representa la enseñanza superior. $Y$ es en este sentido, claramente orientado hacia la planificación docente, en el que se inserta el estudio aquí realizado.

\section{BIBLIOGRAFÍA Y REFERENCIAS DOCUMENTALES}

CLAVE S.L. (1990): La situación urbanística de la Universidad de Sevilla. Sevilla, 321 folios mecanografiados.

CONSEJERÍA DE EDUCACIÓN Y CIENCIA (1993): Octavo Mapa Escolar de Andalucía. Curso 91/92. Sevilla, Junta de Andalucía, 547 páginas.

FERNÁNDEZ ARUFE, J.E. (1982): "Educación y Cultura". Decadencia y Crisis en Andalucía (VELARDE FUERTES, director). Sevilla, Instituto de Desarrollo Regional de la Univ., págs. 879-915.

HERNÁNDEZ, LASSIBILLE y NAVARRO (1993): "La financiación de la Enseñanza Superior en Andalucía: 1987-1993". Revista de Estudios Regionales, n 36. Málaga, Universidades de Andalucía, pgs. 19-50.

INSTITUTO DE ESTADÍSTICA DE ANDALUCÍA (1992): Anuario Estadístico Andalucía 1991. Bilbao, Junta de Andalucía, 822 páginas.

INSTITUTO DE ESTADÍSTICA DE ANDALUCÍA (1994): Andalucía Datos Básicos 1994. Sevilla, Junta de Andalucía, 36 páginas.

INSTITUTO DE ESTADÍSTICA DE ANDALUCÍA (1995): Andalucía Datos Básicos 1995. Sevilla, Junta de Andalucía, 56 páginas.

UNIVERSIDAD DE SEVILLA (1994): "Presupuesto de la Universidad de Sevilla del ejercicio 1994". Boletín de la Universidad de Sevilla, $\mathrm{n}^{\circ}$ 11. Sevilla, Universidad, pgs. 41-48.

UNIVERSIDAD DE SEVILLA (1995): "Informe sobre la cuenta general de liquidación del ejercicio presupuestario de 1993". Boletín de la Universidad de Sevilla, $\mathrm{n}^{\circ}$ 17. Sevilla, Universidad, pgs. 22-27.

UNIVERSIDAD DE SEVILLA (1987): Apertura del curso académico 1986-87. Sevilla, Publicaciones de la Universidad, 74 páginas.

UNIVERSIDAD DE SEVILLA (1988): Apertura del curso académico 1987-88. Sevilla, Universidad, 94 páginas.

VENTURA FERNÁNDEZ, J. (1996): Los servicios educativos en Andalucía: territorio y planificación sectorial. Sevilla, Tesis doctoral inédita de la Hispalense, 769 páginas más anexos. 


\section{APÉNDICE ESTADÍSTICO}

Se incorpora finalmente un apéndice estadístico en el que aparece una base d datos de los municipios de la provincia de Sevilla con las siguientes variables : sus correspondientes descripciones:

- CODIGO: Código estadístico municipal.

- MUNICIPIO: Nombre de cada municipio.

- ALUM_UNISE: Alumnos matriculados en la Universidad en 1994.

- POB_1994: Población total de derecho a 1 de enero de 1994.

- TASA_UNISE: Alumnos Univ. Sev. * 100 / Pob. total derecho.

- TASAENSU91: Alumnos enseñ. sup. * 100 / Pob. > 10 años-1991.

\section{Notas:}

- Por problemas cartográficos no se recogen los datos de los tres último municipios segregados en la provincia de Sevilla: Cañada del Rosal de La Luisiana El Cuervo de Lebrija, y Villafranco del Guadalquivir de Puebla del Río; de ahí qu el número total de alumnos sevillanos en la Hispalense sea aquí inferior en 10 । individuos al que aparece en el Cuadro IV.

- No obstante, la pobIación total de derecho del municipio de Puebla del Rír sí incluye a la de la entidad después segregada (sin que ocurra lo mismo en lo otros dos casos), por lo que en esta ocasión la tasa municipal de alumnos en $1_{\text {i }}$ Hispalense está infravalorada.

- Recordamos que la población total de derecho del municipio de Umbretı hace referencia a 1 de enero de 1993.

CÓDIGO MUNICIPIO

41001 Aguadulce

41002

41003

41004

41005

41006

41007

41008

41009

41010

41011

41012

41013
Alanís

Albaida del Aljarafe

Alcalá de Guadaira

Alcalá del Río

Alcolea del Río

Algaba, La ....

Algámitas

Almadén de la Plata

Almensilla

Arahal

Aznalcázar

Aznalcóllar
ALUM_UNISE POB_1994

TASA_UNISE TASAENSU9

\section{7}

27
39

39

26

1.221

174

43

217

20

44

27

271

53

54
1971

2.114

1.771

54.529

9.414

3.565

12.708

1.469

1.771

2.045

18.249

3.327

5.739
1,36

1,84

1,46

2,23

1,84

1,20

1,70

1,36

2,48

1,32

1,48

1,59

0,94
2,53

1,78

2,35

3,39

2,31

1,78

1,72

1,26

2,37

3,91

2,69

2,43

0,80 
41014

Badolatosa

27

41015 Benacazón

54

41016 Bollullos de la Mitación ........

41017 Bormujos.

41018 Brenes

41019 Burguillos

41020

41021

41022

41023

41024

41025

41026

41027

41028

41029

41030

41031

41032

41033

41034

41035

41036

41037

41038

Cabezas de San Juan, Las ...

Camas

Campana, La

Cantillana

Carmona

Carrión de los Céspedes ........

Casariche

Castilblanco de los Arroyos .

Castilleja de Guzmán

Castilleja de la Cuesta

Castilleja del Campo.

Castillo de las Guardas, El...

Cazalla de la Sierra...

Constantina

Coria del Río

Coripe

Coronil, El

Corrales, Los

41039

Dos Hermanas

41040

Ecija

Espartinas

41041

41042

Estepa

41043

41044

Fuentes de Andalucía.

Garrobo, El

Gelves

41045

Gerena

41046

Gilena

41047

Gines

41048

Guadalcanal

Guillena

41049

Herrera

41051

Huévar

41052

41053

Lantejuela, La

41054

Lebrija

Lora de Estepa

41055

Lora del Río

41056

Luisiana, La

41057

Madroño, El

Mairena del Alcor
107

75

147

32

177

616

53

124

413

48

58

53

89

275

14

18

88

134

375

24

78

23

1.914

576

126

187

148

13

103

73

46

316

48

48

16

41

40

440

6

388

67

3

393

\subsection{4}

4.934

6.209

5.593

10.620

3.374

15.894

25.984

5.470

8.998

24.608

2.363

5.166

4.232

583

15.960

638

1.744

5.235

7.522

23.333

1.709

5.175

4.135

82.14

37.267

3.563

11.325

7.555

748

4.257

5.419

3.824

7.703

3.121

3.121

5.982

2.217

3.539

23.773

741

19.570

4.332

393

15.720
0,84

1,09

1,74

1,34

1,38

0,94

1,11

2,38

0,96

1,37

1,67

2,03

1,12

1,25

15,26

1,72

2,19

1,03

1,68

1,78

1,60

1,40

1,50

0,55

2,31

1,54

3,53

1,64

1,95

1,73

2,41

1,34

1,20

4,10

1,53

1,51

0,26

1,84

1.13

1.85

0.80

1.98

1.54

0.76

2.50
1,50

2,17

2,82

2,47

1,50

1,23

1,10

3,50

1,60

2,64

3,66

2,53

1,71

1,67

10,58

3,71

4,20

2,32

2,68

3,33

2,70

2,71

2,41

1,68

4,76

3,91

7,38

3,80

2,32

1,09

4,98

2,16

2,52

9,92

2,54

1,42

2,77

2,44

0.81

2.22

2.16

2.36

1.26

1.59

4.19 


\begin{tabular}{|c|c|c|c|c|c|}
\hline CÓDIGO & MUNICIPIO & ALUM_UNISE & POB_1994 & TASA_UNISE & TASAENSU9 \\
\hline 41059 & Mairena del Aljarafe ............... & 1.023 & 28.551 & 3.58 & 9.48 \\
\hline 41060 & 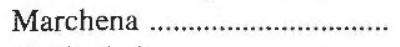 & 424 & 17.823 & 2.37 & 3.71 \\
\hline 41061 & 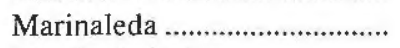 & 20 & 2.617 & 0.76 & 1.13 \\
\hline 41062 & Martín de la Jara ..................... & 25 & 2.838 & 0.88 & 1.31 \\
\hline 41063 & 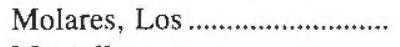 & 35 & 2.780 & 1.25 & 2.23 \\
\hline 41064 & 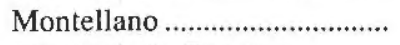 & 72 & 7.046 & 1.02 & 1.82 \\
\hline 41065 & Morón de la Frontera .............. & 482 & 29.408 & 1.63 & 2.95 \\
\hline 41066 & Navas de la Concepción, Las & 30 & 2.008 & 1.49 & 1.57 \\
\hline 41067 & 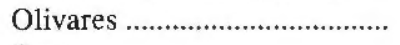 & 103 & 7.093 & 1.45 & 2.99 \\
\hline 41068 & 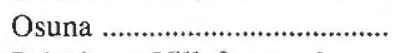 & 302 & 17.109 & 1.76 & 4.47 \\
\hline 41069 & Palacios y Villafranca, Los... & 371 & 31.210 & 1.18 & 1.63 \\
\hline 41070 & Palomares del Río .................... & 91 & 2.946 & 3.08 & 8.49 \\
\hline 41071 & 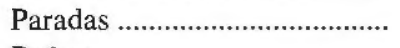 & 113 & 7.187 & 1.57 & 2.24 \\
\hline 41072 & Pedrera & 60 & 4.898 & 1.22 & 1.44 \\
\hline 41073 & 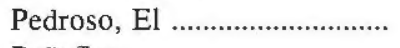 & 38 & 2.445 & 1.55 & 2.39 \\
\hline 41074 & 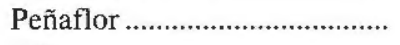 & 10 & 4.092 & 0.24 & 2.96 \\
\hline 41075 & 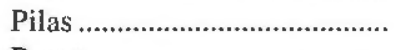 & 199 & 11.042 & 1.80 & 2.79 \\
\hline 41076 & 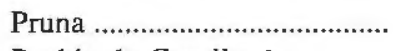 & 38 & 3.485 & 1.09 & 1.55 \\
\hline 41077 & Puebla de Cazalla, La ............ & 146 & 11.133 & 1.31 & 1.97 \\
\hline 41078 & Puebla de los Infantes, La.... & 54 & 3.649 & 1.48 & 2.55 \\
\hline 41079 & Puebla del Río, La ................... & 268 & 16.976 & 1.57 & 2.11 \\
\hline 41080 & 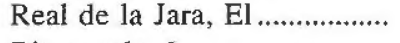 & 20 & 1.745 & 1.14 & 2.80 \\
\hline 41081 & Rinconada, La ......................... & 393 & 23.849 & 1.64 & 1.72 \\
\hline 41082 & Roda de Andalucía, La .......... & 29 & 4.139 & 0.70 & 2.66 \\
\hline 41083 & 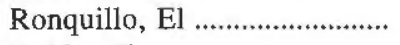 & 23 & 1.373 & 1.69 & 1.97 \\
\hline 41084 & 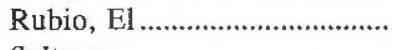 & 66 & 3.687 & 1.79 & 3.14 \\
\hline 41085 & 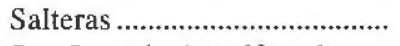 & 71 & 2.712 & 2.61 & 3.47 \\
\hline 41086 & San Juan de Aznalfarache .... & 549 & 23.258 & 2.36 & 3.20 \\
\hline 41087 & Sanlúcar la Mayor ..................... & 223 & 10.047 & 2.21 & 4.51 \\
\hline 41088 & San Nicolás del Puerto ........... & 6 & 717 & 0.83 & 1.58 \\
\hline 41089 & Santiponce ............................ & 98 & 6.557 & 1.49 & 1.82 \\
\hline 41090 & 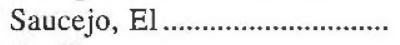 & 43 & 4.144 & 1.03 & 2.39 \\
\hline 41091 & Sevilla & 30.636 & 714.148 & 4.28 & 9.65 \\
\hline 41092 & 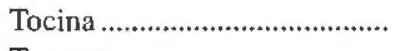 & 120 & 8.977 & 1.33 & 2.02 \\
\hline 41093 & 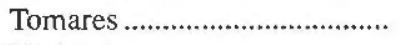 & 665 & 15.007 & 4.43 & 14.70 \\
\hline 41094 & Umbrete & 85 & 3.014 & 2.82 & 2.37 \\
\hline 41095 & Utrera & 810 & 45.008 & 1.79 & 3.02 \\
\hline 41096 & Valencina de la Concepción. & 183 & 5.038 & 3.63 & 7.32 \\
\hline 41097 & Villamanrique de la Condesa & 41 & 3.668 & 1.11 & 1.62 \\
\hline 41098 & Villanueva del Ariscal ............ & 80 & 4.172 & 1.91 & 4.13 \\
\hline 41099 & Villanueva del Río y Minas . & 47 & 6.201 & 0.75 & 1.71 \\
\hline 41101 & Villaverde del Río ..................... & 95 & 6.765 & 1.40 & 2.21 \\
\hline 41102 & Viso del Alcor, El ...................... & 224 & 15.600 & 1.43 & 1.98 \\
\hline
\end{tabular}

